\title{
Extended Duration Dual Antiplatelet Therapy After Percutaneous Coronary Intervention in Patients With Peripheral Arterial Disease: A Meta-Analysis
}

\author{
Hua Ling ${ }^{\mathrm{a}, \mathrm{e}}$, Ebony Andrews ${ }^{\mathrm{b}}$, David Ombengic ${ }^{\mathrm{c}}$, Fang Li ${ }^{\mathrm{d}}$
}

\begin{abstract}
Background: Patients with peripheral arterial disease (PAD) undergoing percutaneous coronary intervention (PCI) are at elevated risk of ischemic and bleeding events. However, the optimal duration of dual antiplatelet therapy (DAPT) after PCI in patients with PAD remains unclear.
\end{abstract}

Methods: A systematic literature search was performed through June 2017 using PubMed, EMBASE and Cochrane databases with the following key terms: "dual antiplatelet therapy", "P2Y12 inhibitor", "myocardial infarction", "percutaneous coronary intervention", "stent", "peripheral arterial disease", and "ankle-brachial index". The analysis was restricted to randomized trials published in English in patients with PAD receiving extended DAPT (> 12-month) after PCI. Overall analysis was performed using Review Manager 5.3 with the Mantel-Haenszel method.

Results: Two randomized controlled trials involving 895 patients were included in this review. Compared to the placebo group, there was no statistical significance in the occurrence of major adverse cardiovascular and cerebrovascular events (MACCE) in patients receiving extended DAPT (odds ratio (OR) 0.76, 95\% confidence interval (CI) $0.37-1.57 ; \mathrm{P}=0.46)$. The results were associated with substantial heterogeneity $\left(\mathrm{I}^{2}=71 \%, \mathrm{P}=0.07\right)$. Extended DAPT was not significantly associated with increased moderate/severe bleeding events (OR 1.63, 95\% CI $0.84-3.18 ; \mathrm{P}=0.15 ; \mathrm{I}^{2}=0 \%, \mathrm{P}=0.59$ ). The extended DAPT was associated with $82 \%$ relative risk reduction in the events of definite/probably stent thrombosis.

Conclusions: Among patients with PAD, extended DAPT after PCI

Manuscript submitted March 29, 2018, accepted April 11, 2018

aDepartment of Pharmacy Practice, School of Pharmacy, Georgia CampusPhiladelphia College of Osteopathic Medicine, Suwanee, GA, USA

${ }^{b}$ Department of Pharmacy Practice, School of Pharmacy, Hampton University, Hampton, VA, USA

'Department of Clinical Sciences, Medical College of Wisconsin Pharmacy School, Milwaukee, WI, USA

dJiangsu Vocational College of Medicine, Yancheng, Jiangsu, China

${ }^{e}$ Corresponding Author: Hua Ling, School of Pharmacy, Georgia CampusPhiladelphia College of Osteopathic Medicine, 625 Old Peachtree Rd NW, Suwanee, GA 30024, USA. Email: hua.ling@outlook.com

doi: https://doi.org/10.14740/cr719w resulted in a non-significant difference in ischemic and bleeding events compared to placebo, respectively. The routine use of extended DAPT in this cohort should be carefully evaluated.

Keywords: DAPT, PCI, PAD

\section{Introduction}

Peripheral arterial disease (PAD) is associated with more extensive and calcified coronary atherosclerosis, constrictive arterial remodeling, and greater disease progression in patients with coronary artery disease, thus contributing to adverse cardiovascular outcomes [1]. It is estimated that PAD affects approximately 8.5 million people in the United States [2]. It is reported that two out of three patients with PAD had coexistent coronary arterial disease (CAD) and one in five patients with CAD had coexistent PAD $[3,4]$. Patients with PAD undergoing percutaneous coronary intervention (PCI) are at elevated risk of adverse clinical events. The data from the Dutch Stent Thrombosis Registry found that PAD was an independent risk factor associated with 5.62-fold increased odds of late (>30 days) stent thrombosis [5]. Patients with PAD were also reported to have a 2-fold increase risk of death and a trend toward higher major bleeding complications after PCI $[4,6]$. Dual antiplatelet therapy (DAPT) with aspirin and a $\mathrm{P} 2 \mathrm{Y}_{12}$ inhibitor after $\mathrm{PCI}$ is generally recommended to reduce the risk of stent thrombosis and ischemic events, which unfortunately may be offset by the increased risk of bleeding [7]. The optimal duration of DAPT has been debated in the past few decades and decisions regarding duration of DAPT require a comprehensive assessment of the benefits of treatment, bleeding risk, and patient preference. In the PEGASUS-TIMI 54 (Prevention of Cardiovascular Events in Patients With Prior Heart Attack Using Ticagrelor Compared to Placebo on a Background of Aspirin-Thrombolysis In Myocardial Infarction 54) trial, DAPT with a median treatment duration of 33 months achieved approximately $40 \%$ reduction in cardiovascular death, myocardial infarction (MI), or stroke in patients with PAD, compared to patients without PAD [6]. A non-significant increase of Thrombolysis in Myocardial Infarction (TIMI) major or minor bleeding was observed in patients with PAD treated with DAPT 


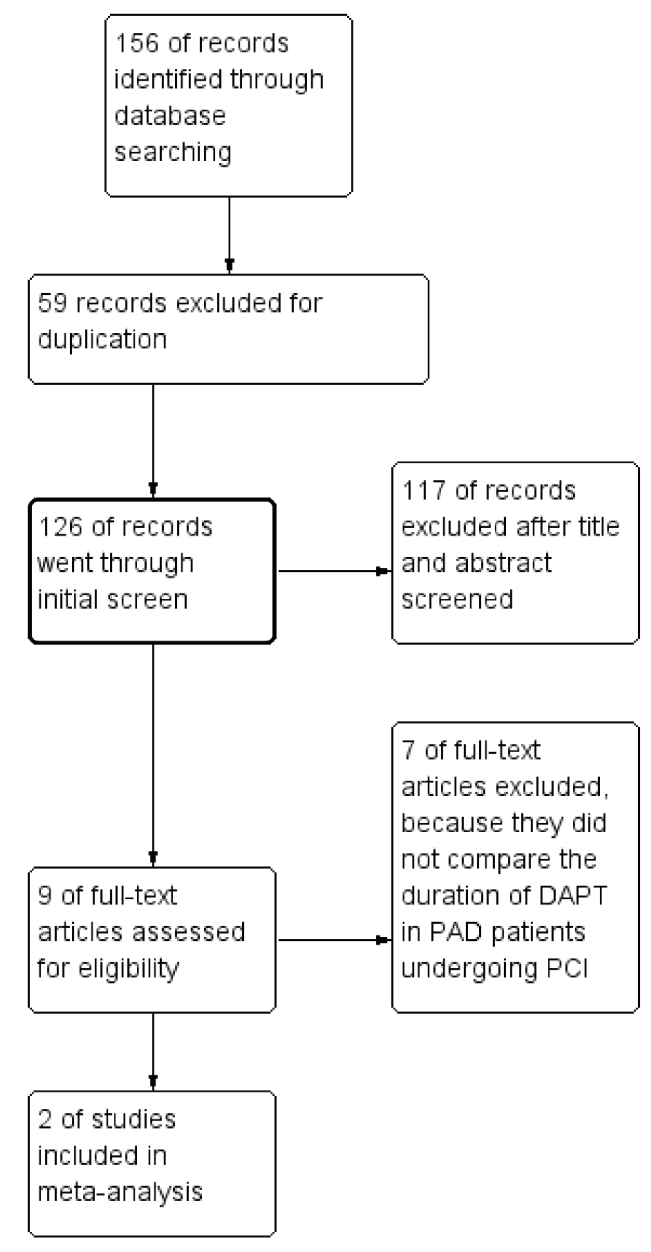

Figure 1. Summary of the study selection and exclusion process.

in the PEGASUS-TIMI 54 trial. Similar findings have been reported in the CHARISMA (Clopidogrel for High Atherothrombotic Risk and Ischemic Stabilization, Management, and Avoidance) trial with $40 \%$ reduction in MI in patients received DAPT for 28 months [8]. That said, patients with PAD in CHARISMA trial experienced almost $70 \%$ increase of minor bleeding compared to aspirin monotherapy $(20.4 \%$ vs. $34.4 \%$ ). Considering the elevated risk of ischemic and bleeding events in patients with PAD undergoing PCI, it is uncertain about whether DAPT should be extended beyond 12 months based on the benefit-risk profile. We conducted a meta-analysis on this topic to better understand the benefits and risks of DAPT beyond 12-month for secondary prevention in patients with PAD undergoing PCI.

\section{Methods}

The studies included in the meta-analysis to evaluate the duration of DAPT in patients with PAD undergoing PCI were identified by conducting a systematic review of literature indexed in database PubMed, EMBASE and Cochrane databases.
Key words used to conduct the search were "dual antiplatelet therapy", "P2Y 12 inhibitor", "myocardial infarction", "percutaneous coronary intervention", "stent", "peripheral arterial disease", and "ankle-brachial index". The literature search was performed through June 2017. In addition, published conference abstracts, review articles, and editorials were reviewed for further information.

The meta-analysis included original research that met the following inclusion criteria: 1) randomized controlled study as design; 2) published in English; 3) conducted in patients with PAD undergoing coronary stenting with either drug-eluting stents (DES) or bare metal stents; and 4) compared the efficacy of extended DAPT (> 12-month) vs. non-extended DAPT ( $\leq$ 12-month). The exclusion criteria were as follows: 1) studies whose analysis did not include patients with PAD;2) studies that did not have controls.

The primary endpoint for this analysis was the incidence of MACCE, defined as a composite of all-cause death, MI, and stroke. Incidence of GUSTO (the Global Use of Strategies to Open Occluded Coronary Arteries) moderate/severe bleeding was used for the safety endpoint. Events of definite or probable stent thrombosis, MI, cardiac death, and all-cause death were also evaluated.

This meta-analysis followed the guidelines of the PRISMA Statement 2009 [9]. The two authors independently assessed the eligibility of trials and extracted the data. Disagreements were resolved by consensus. The Cochrane Risk of Bias Tool was used to assess bias risk. All statistical analyses were performed by Review Manager 5.3 (Nordic Cochrane Centre, Cochrane Collaboration) using the Mantel-Haenszel method with either random effects or fixed effects models based on the level of heterogeneity. Odds ratio (OR) of efficacy and safety end points were calculated from the included studies in patients with PAD undergoing PCI receiving extended vs. non-extended DAPT. We tested heterogeneity of the included studies with Cochran Q statistics and the extent of inconsistency between results with $\mathrm{I}^{2}$ statistics with significance set at a $P$ value of 0.10 . Data are presented as OR with $95 \%$ confidence intervals $(\mathrm{CI})$, with two-tailed $\mathrm{P}$ values and statistical significance set at $P$ value $<0.05$.

\section{Results}

Overall, 185 citations met the search criteria to be assessed. After deleting 59 duplications, 126 citations were reviewed in detail for eligibility. Most citations were excluded because they were review articles, abstracts, editorials, non-randomized registry studies, or not relevant to our topic (Fig. 1). Particularly, the CHARISMA trial was excluded because it compared DAPT vs. aspirin monotherapy in patients with PAD [8]. The PEGASUS-TIMI 54 trial was excluded because it included patients with MI that occurred 1 - 3 years prior to enrollment [6]. Furthermore, patients in PEGASUS-TIMI 54 trial were also not required to have a history of PCI with stenting.

After exclusion, two randomized controlled trials involving 895 patients were included in this analysis. The main description of both studies and the participants' characteristics 
Table 1. Summary of the DAPT Trial and PRODIGY Trial

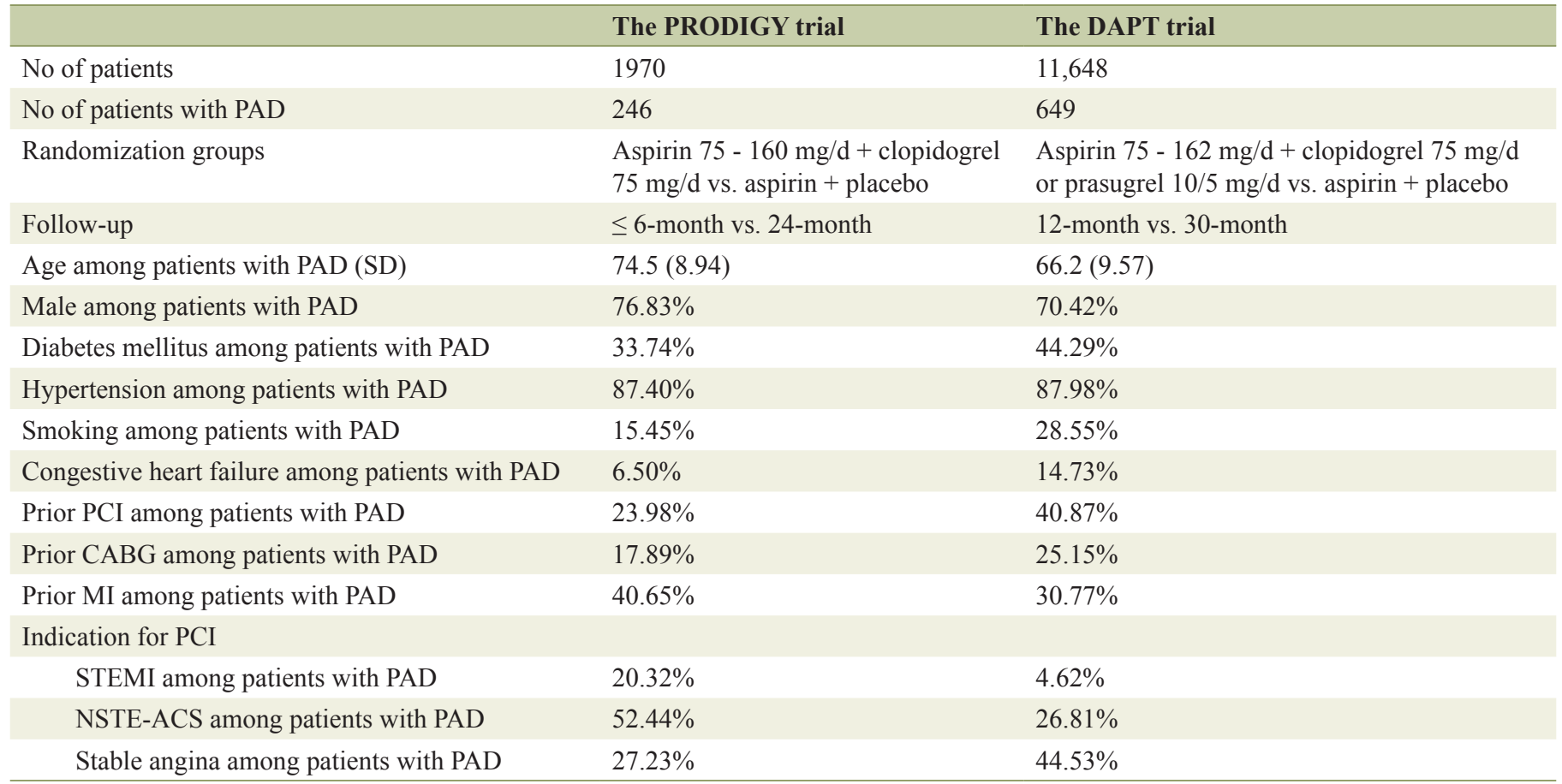

CABG: coronary artery bypass grafting; MI: myocardial infarction; PAD: peripheral artery disease; SD: standard deviation.

are listed in Table 1. The PRODIGY (Prolonging Dual Antiplatelet Treatment After Grading Stent-Induced Intimal Hyperplasia Study) trial was a randomized, multicenter, openlabel study, and the subanalysis of PRODIGY trial assessed the efficacy and safety of prolonged (24-month) vs. short ( $\leq$ 6-month) DAPT (clopidogrel plus aspirin) in 246 and 1,724 patients with and without PAD undergoing PCI, respectively [10]. The DAPT trial was a randomized, double-blind, placebo-controlled trial, and the subanalysis of the DAPT trial evaluated the efficacy of extended DAPT (clopidogrel or prasugrel plus aspirin; 30-month vs. 12-month) in reducing ischemic events and the safety profile of extended DAPT in 10,999 non-PAD vs. 649 PAD patients [11]. Of note, approximately half participants in both the PRODIGY and the DAPT trial received everolimus-eluting stent or zotarolimus-eluting stent, which are considered as new-generation DES with less risk of stent thrombosis.

The incidence of MACCE was $12.8 \%$ (56/437 patients) when receiving extended DAPT vs. $15.5 \%$ (71/458 patients) in patients received non-extended DAPT, representing a nonsignificant relative risk reduction $(\mathrm{OR}=0.76$; 95\% CI 0.37 -
1.57; $\mathrm{P}=0.46$ ) (Fig. 2). The Chi-square test for heterogeneity was significant at a level of less than $0.10(\mathrm{P}=0.07)$. The $\mathrm{I}^{2}$ value was $70 \%$. These quantitative results suggest there was study variability.

The occurrence of GUSTO moderate/server bleeding was $5.0 \%$ (22/437 patients) when receiving extended DAPT vs. $3.1 \%(14 / 458$ patients) when receiving non-extended DAPT $(\mathrm{OR}=1.67 ; 95 \%$ CI 0.84 - 3.32; P=0.14) (Fig. 3). Extended DAPT was not significantly associated with increased GUSTO moderate/severe bleeding events, without heterogeneity between studies.

The extended DAPT was associated with $82 \%$ relative risk reduction in the events of definite/probably stent thrombosis, compare to the non-extended DAPT (OR $=0.18 ; 95 \%$ CI $0.05-0.68 ; \mathrm{P}=0.01$ ) (Fig. 4). The risk reduction represented an absolute risk difference of $2.60 \%$ or a number needed to treat of 38 to prevent one stent thrombosis over an extended duration of DAPT. However, this significant reduction in stent thrombosis was not accompanied by any significant reduction in MI, cardiac death, or all-cause death. (Fig. 5-7) Though there was little heterogeneity in the analysis of stent thrombo-

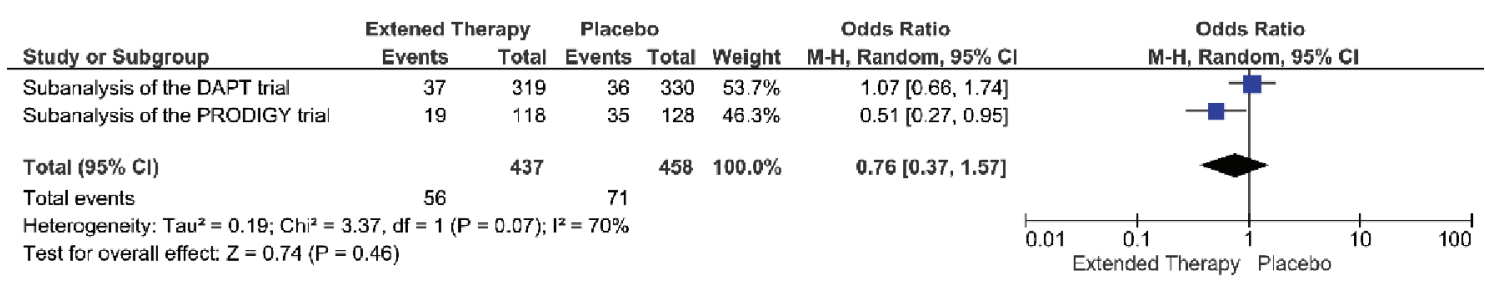

Figure 2. MACCE associated with a short versus extended DAPT use in patients with PAD after PCI. 


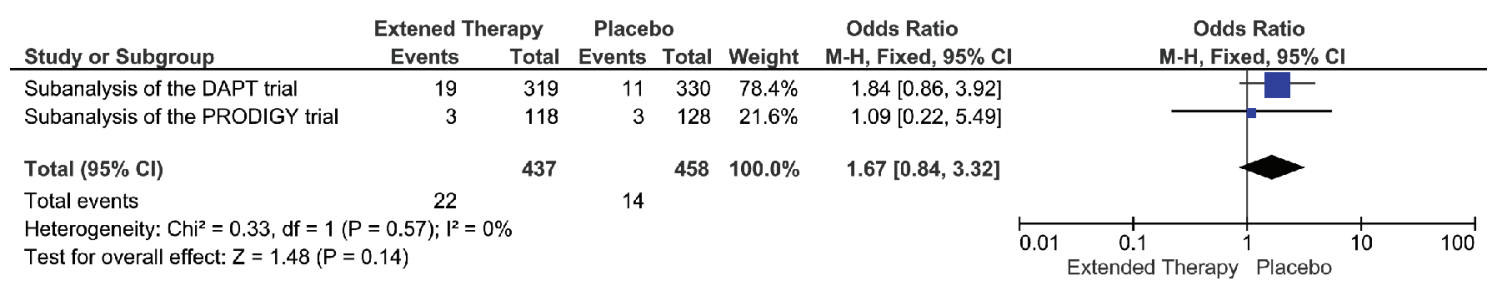

Figure 3. GUSTO moderate/severe bleeding events associated with a short versus extended DAPT use in patients with PAD after PCl.

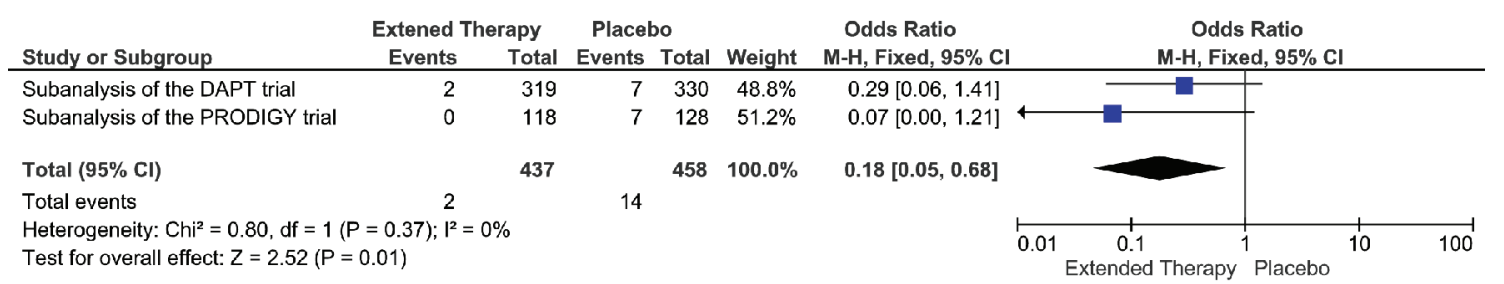

Figure 4. Definite or probable stent thrombosis events associated with a short versus extended DAPT use in patients with PAD after PCl.

\begin{tabular}{|c|c|c|c|c|c|c|c|c|c|c|}
\hline \multirow[b]{2}{*}{ Study or Subgroup } & \multicolumn{2}{|c|}{ Extened Therapy } & \multicolumn{2}{|c|}{ Placebo } & \multicolumn{3}{|c|}{ Odds Ratio } & \multirow{2}{*}{\multicolumn{2}{|c|}{$\begin{array}{c}\text { Odds Ratio } \\
\text { M-H, Fixed, } 95 \% \text { Cl }\end{array}$}} & \\
\hline & Events & Total & Events & Total & Weight & M-H, Fixed, $95 \% \mathrm{Cl}$ & & & & \\
\hline Subanalysis of the DAPT trial & 14 & 319 & 22 & 330 & $67.6 \%$ & $0.64[0.32,1.28]$ & & & & \\
\hline Subanalysis of the PRODIGY trial & 7 & 118 & 11 & 128 & $32.4 \%$ & $0.67[0.25,1.79]$ & & & & \\
\hline Total $(95 \% \mathrm{Cl})$ & & 437 & & 458 & $100.0 \%$ & $0.65[0.37,1.15]$ & & & & \\
\hline Total events & 21 & & 33 & & & & & & & \\
\hline $\begin{array}{l}\text { Heterogeneity: } \mathrm{Chi}^{2}=0.00, \mathrm{df}=1 \\
\text { Test for overall effect: } \mathrm{Z}=1.49(\mathrm{P}\end{array}$ & $\begin{array}{l}=0.94) ; 1^{2}= \\
0.14)\end{array}$ & & & & & & 0.01 & $\begin{array}{c}0.1 \\
\text { nded }\end{array}$ & 10 & 100 \\
\hline
\end{tabular}

Figure 5. Ml events associated with a short versus extended DAPT use in patients with PAD after PCl.

sis and MI, the analysis of cardiac death and all-cause death were associated with substantial heterogeneity, indicating study variability.

The risk of bias in included studies was low or unclear in most areas in both studies (Fig. 8). The performance bias and attrition bias were the main potential sources of bias in the PRODIGY trial. The open-label design of the PRODIGY trial may have introduced a potential for performance bias, and more patients in the short-term arm were reported to discontinue clopidogrel after the first month (12\% vs. $0.2 \%)$, which was allowed in patients who were randomized to a BMS. However, the discontinuation rate was not reported in the PAD population, so it is unclear about attrition bias in the subgroup analysis of the PRODIGY trial.

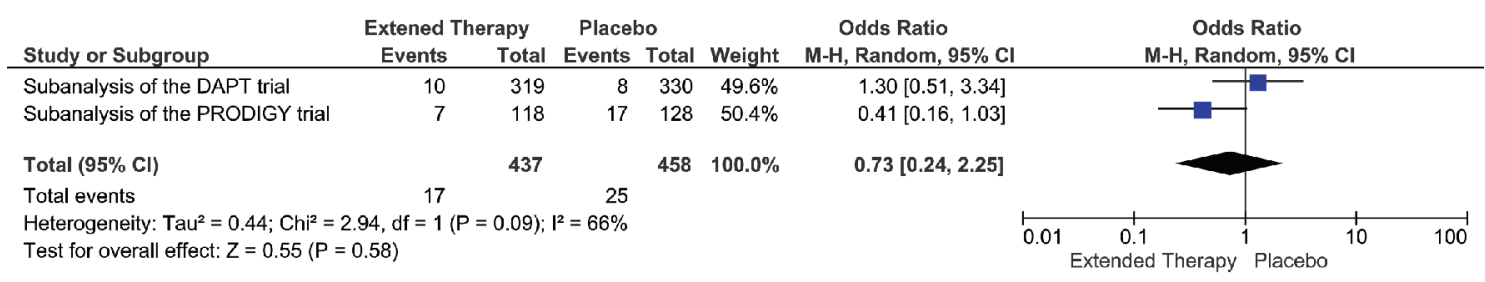

Figure 6. Cardiac death events associated with a short versus extended DAPT use in patients with PAD after PCI.

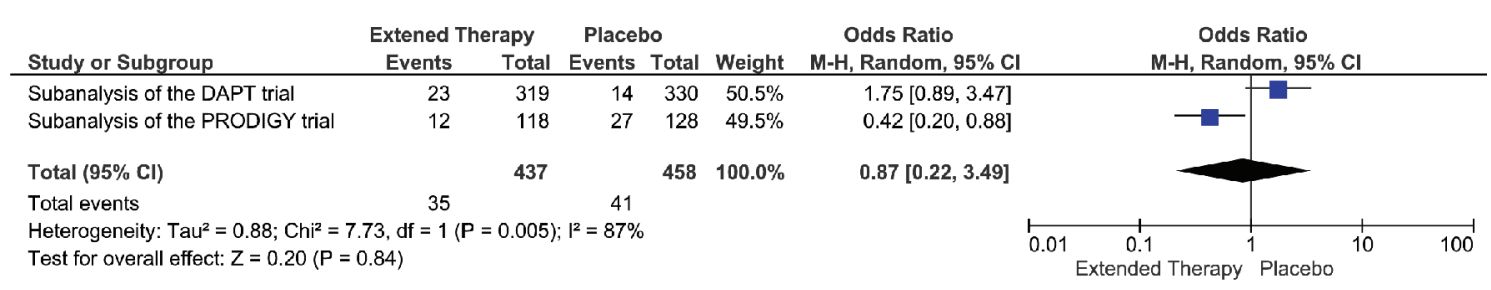

Figure 7. All-cause death events associated with a short versus extended DAPT use in patients with PAD after PCI. 


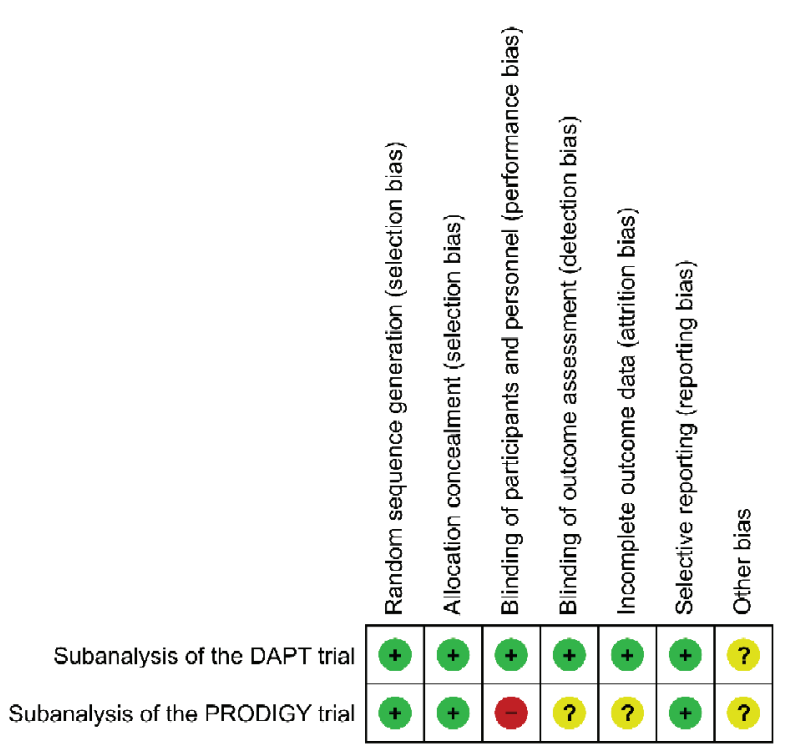

Figure 8. Risk of bias graph for the studies included in this systematic review. Green +: yes (low risk of bias); yellow ?: unclear; red -: no (high risk of bias).

\section{Discussion}

Our meta-analysis of two randomized controlled trials involving 895 patients with PAD undergoing PCI found a non-statistically significant reduction of MACCE in patients receiving extended DAPT. The extended duration of DAPT was not significantly associated with increased GUSTO moderate/severe bleeding events either. A substantial reduction of definite/ probably stent thrombosis was observed in the extended DAPT group. Unfortunately, the reduction of stent thrombosis alone was not able to reduce the occurrence of MI, cardiac death, and all-cause death significantly. The analysis of MACCE, cardiac death, and all-cause death were associated with substantial heterogeneity.

The subanalysis of the DAPT trial and PRODIGY trial found that PAD was associated with increased risk of ischemic and bleeding events after PCI, which were consistent with the previous findings in the CHARISMA trial and PEGAUSIS-TIMI 54 trial. In general, the PAD patients were usually older and more likely to have hypertension, MI, multiple stents, and diabetes mellitus. In the DAPT trial and PRODIGY trial, patients with PAD were associated with higher rates of stent thrombosis, MI, MACCE compared with patients without PAD, indicating that PAD is a risk factor of adverse health outcomes (Table 2). The incidence of GUSTO moderate/severe bleeding was significantly higher in patients with PAD compared with patients without PAD in the DAPT trial, though the rate of bleeding-related death was similar [11]. Interestingly, the increased rate of GUSTO moderate/ severe bleeding was not observed in the PRODIGY trial, which could be explained by the small sample size and inadequate power [10]. Due to the evaluated risk of ischemic and bleeding events in patients with PAD, it will be necessary to investigate if the duration of DAPT should be tailored based on the status of PAD.

Current guidelines recommend at least 12-month treatment of DAPT in patients following MI, however, the duration of DAPT has been debated since the beginning of DAPT [7]. The DAPT trial found reduced ischemic event and increased bleeding, thus suggesting the extended duration could be used in patients at high ischemic patients with low bleeding risk [12]. Subsequently, subgroup studies have been conducted to define adequate patients for use of extended DAPT therapy. However, to the best of our knowledge, very few trials have been conducted in patients with PAD, which were also limited by small sample size. By using meta-analysis, we intended to combine the limited results together to advance our understanding the role of extended DAPT in patients with PAD undergoing PCI.

This meta-analysis did not detect a significant reduction in MACCE, cardiac death and all-cause death in extended DAPT group in patients with PAD after PCI. It seems that the elevated all-cause death rate in the extended DAPT group is not linked to the increased bleeding rate [13]. A post-hoc analysis of the DAPT trial identified a significant difference of cancer related deaths in both groups $(0.6 \%$ vs. $0.3 \%, P=0.02)$ [13]. After adjusting the cancer incidence, a sensitive analysis revealed a non-significant death rate in both groups, suggesting that the non-significant reduction of MACCE and all-cause death may be explained by the imbalanced baseline characteristics in both groups [13]. In addition, the duration of non-extended DAPT in the PRODIGY trial was only 6 month [14]. For 50\% of the patients in the PRODIGY trial who received bare mental stent and first-generation DES, it is recommended to receive DAPT at least 12-month. The new-generation stents have been tested in the non-ACS patients showing non-inferior effectiveness in 6-month vs. 12-month [15]. Nevertheless, the duration

Table 2. Effect of PAD on Clinical Outcomes in the DAPT Trial and PRODIGY Trial

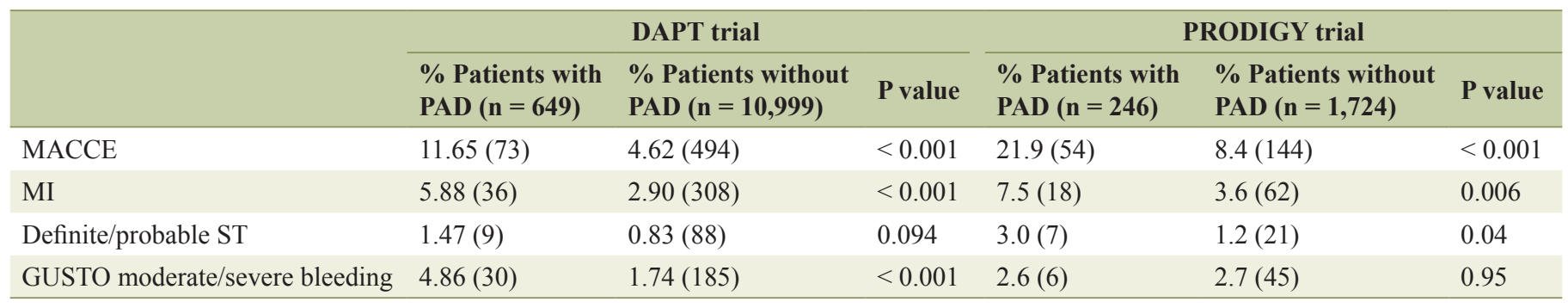

ST: stent thrombosis; MACCE: major adverse cardiovascular and cerebrovascular event; MI: myocardial infarction. 
of DAPT in ACS patients with new-generation stent has not been fully examined. Therefore, the increased incidence of MACCE and cardiac-death in the non-extended DAPT group could be attributed to the increased stent thrombosis events because of insufficient protection from the short duration DAPT (6-month).

The GUSTO moderate and severe bleeding risk was not significantly increased in the extended DAPT group in our meta-analysis. This result is consistent with a recent metaanalysis of 21,534 participants from three trials of post-MI patients received DAPT beyond 12-month [16]. The odds ratio of major bleeding and fatal bleeding were $1.53(0.92,2.52)$ and $1.12(0.66,1.90)$ with both $\mathrm{P}$ values higher than $0.05 .(\mathrm{P}=0.10$ and $\mathrm{P}=0.69)$. In the PEGASUS-TIMI 54 trial subanalysis, neither TIMI major nor minor bleeding was associated with extended DAPT in patients with PAD [6]. In the CHARISMA trial subanalysis, there was no significant difference in the rate of severe, fatal, or moderate bleeding, though minor bleeding was reported to increase almost 2-fold in the extended DAPT group in the CHARISMA trial [8].

There were high levels of heterogeneity in the analyses of MACCE, cardiac death and all-cause death, which could be explained by the differences between the two trials in treatment lengths and baseline characteristics of study participants, though little heterogeneity was observed in stent thrombosis, MI, and GUSTO moderate/severe bleeding. Participants in the PRODIGY trial were more likely to have older age and previous MI. It was reported that more than 50\% participants had acute $\mathrm{MI}$ at presentation in the PRODIGY trial, and less than $25 \%$ patients had stable CAD compared to $45 \%$ patients with stable CAD in the DAPT trial. Hence, patients in the PRODIGY trial presented more acute conditions, and thereby might benefit more from the extended DAPT. Also as aforementioned, there was an imbalance of cancer in the baseline characteristics between groups in the DAPT trial, potentially increased the all-cause death rate in the treatment group. Furthermore, the duration of non-extended DAPT group was 6-month in PRODIGY trial vs. 12-month in the DAPT trial. Patients in the non-extended DAPT group (6-month) of PRODIGY trial was associated with significant increase in MACCE and a non-significant change of definite or probable stent thrombosis, indicating short duration DAPT (6-month) may not be able to provide enough protection in ACS patients with a comorbidity of PAD receiving new-generation DES, causing overestimation of the benefits of extended DAPT in MACCE in our results.

There are certain limitations in our meta-analysis. Although we have conducted a comprehensive search by two persons independently, only two randomized clinical trials were eligible and included in our study. Theoretically, two trials are the minimal requirement to conduct a meta-analysis, and more studies provide a more precise estimation of the true effect than each trial alone. To achieve valid statistical inferences, the random-effects method was used to combine treatment effects when the presence of between-trial heterogeneity is suspected. The combination of only a few studies is not uncommon, especially in cases of rare diseases [17]. In an analysis of 22,453 meta-analyses from the Cochrane Database of Systematic Reviews, more than one third of the meta- analyses included the minimum requirement of two studies only, and close to $75 \%$ meta-analyses contained five or fewer studies [18]. Nevertheless, the meta-analysis of only two studies in the presence of heterogeneity may be considered an unsolved problem and beyond the scope of this study [19]. We acknowledge that the low number of studies makes it difficult to estimate the between-studies variance in random-effects analysis, and some of our results should be interpreted with caution. Moreover, procedural factors of the initial PCI play a major role in determining the risk of ischemic events. For example, it must be noted that substantial patients received new-generation DES in both trials, however, the percentage of participants with new-generation DES was not reported in their subanalysis. New-generation of DES has been reported with $50 \%$ less risk of stent thrombosis compared with the firstgeneration stent. Indeed, the recent guidelines suggested that 6-month DAPT is reasonable after new-generation DES implantation in patients with stable CAD. Unfortunately, because of the nature of our data, we had no access to patients' specific data thus were not able to identify if the percentage of newgeneration DES implantation will affect our results.

\section{Conclusions}

Patients with PAD undergoing PCI are at elevated risk of cardiovascular morbidity and mortality due to increased ischemic and bleeding events. Among patients with PAD, extended DAPT after PCI resulted in a non-significant difference in MACCE, cardiac death, all-cause death and bleeding events and a significant reduction in stent thrombosis and MI compared to placebo. The review was limited by the small number of published trials and high level of heterogeneity in MACCE, cardiac death and all-cause death. Our results showed that the routine use of extended DAPT in patients with PAD undergoing PCI provided a neutral effect on cardiac outcomes, and further studies with adequate power are needed to substantiate the role of extended DAPT in this cohort, especially with newgeneration DES.

\section{Author Contributions}

HL, EA, DO designed the study and acquired, analyzed, and interpreted data. DA, DO, and FL did the literature search and study selection procedures. HL drafted the manuscript, with critical revisions for important intellectual content from all authors. All authors read and approved the final manuscript.

\section{Conflict of Interest}

The authors declare that they have no competing interest.

\section{Funding}

Not applicable. 


\section{Declarations}

All data generated or analyzed during this study are included in this published article.

\section{Abbreviations}

CAD: coronary arterial disease; DAPT: dual antiplatelet therapy; DES: drug-eluting stents; GUSTO: the Global Use of Strategies to Open Occluded Coronary Arteries; MACCE: Major Adverse Cardiovascular and Cerebrovascular Events; MI: myocardial infarction; PAD: peripheral arterial disease; PCI: percutaneous coronary intervention; PEGASUS-TIMI 54: Prevention of Cardiovascular Events in Patients With Prior Heart Attack Using Ticagrelor Compared to Placebo on a Background of Aspirin-Thrombolysis In Myocardial Infarction 54; PRODIGY: Prolonging dual antiplatelet treatment after grading stent-induced intimal hyperplasia study; TIMI: Thrombolysis in Myocardial Infarction

\section{Reference}

1. Hussein AA, Uno K, Wolski K, Kapadia S, Schoenhagen P, Tuzcu EM, Nissen SE, et al. Peripheral arterial disease and progression of coronary atherosclerosis. J Am Coll Cardiol. 2011;57(10):1220-1225.

2. Allison MA, Ho E, Denenberg JO, Langer RD, Newman AB, Fabsitz RR, Criqui MH. Ethnic-specific prevalence of peripheral arterial disease in the United States. Am J Prev Med. 2007;32(4):328-333.

3. Fowkes FG, Low LP, Tuta S, Kozak J, Investigators A. Ankle-brachial index and extent of atherothrombosis in 8891 patients with or at risk of vascular disease: results of the international AGATHA study. Eur Heart J. 2006;27(15):1861-1867.

4. Saw J, Bhatt DL, Moliterno DJ, Brener SJ, Steinhubl SR, Lincoff AM, Tcheng JE, et al. The influence of peripheral arterial disease on outcomes: a pooled analysis of mortality in eight large randomized percutaneous coronary intervention trials. J Am Coll Cardiol. 2006;48(8):1567-1572.

5. van Werkum JW, Heestermans AA, Zomer AC, Kelder JC, Suttorp MJ, Rensing BJ, Koolen JJ, et al. Predictors of coronary stent thrombosis: the Dutch Stent Thrombosis Registry. J Am Coll Cardiol. 2009;53(16):1399-1409.

6. Bonaca MP, Bhatt DL, Storey RF, Steg PG, Cohen M, Kuder J, Goodrich E, et al. Ticagrelor for prevention of ischemic events after myocardial infarction in patients with peripheral artery disease. J Am Coll Cardiol. 2016;67(23):2719-2728.

7. Levine GN, Bates ER, Bittl JA, Brindis RG, Fihn SD, Fleisher LA, Granger CB, et al. 2016 ACC/AHA guideline focused update on duration of dual antiplatelet therapy in patients with coronary artery disease: A report of the American College of Cardiology/American Heart Association Task Force on Clinical Practice Guidelines. J Thorac Cardiovasc Surg. 2016;152(5):1243-1275.
8. Cacoub PP, Bhatt DL, Steg PG, Topol EJ, Creager MA, Investigators $\mathrm{C}$. Patients with peripheral arterial disease in the CHARISMA trial. Eur Heart J. 2009;30(2):192-201.

9. Liberati A, Altman DG, Tetzlaff J, Mulrow C, Gotzsche PC, Ioannidis JP, Clarke M, et al. The PRISMA statement for reporting systematic reviews and meta-analyses of studies that evaluate healthcare interventions: explanation and elaboration. BMJ. 2009;339:b2700.

10. Franzone A, Piccolo R, Gargiulo G, Ariotti S, Marino M, Santucci A, Baldo A, et al. Prolonged vs short duration of dual antiplatelet therapy after percutaneous coronary intervention in patients with or without peripheral arterial disease: a subgroup analysis of the PRODIGY randomized clinical trial. JAMA Cardiol. 2016;1(7):795-803.

11. Secemsky EA, Yeh RW, Kereiakes DJ, Cutlip DE, Steg PG, Massaro JM, Apruzzese PK, et al. Extended duration dual antiplatelet therapy after coronary stenting among patients with peripheral arterial disease: a subanalysis of the dual antiplatelet therapy study. JACC Cardiovasc Interv. 2017;10(9):942-954.

12. Mauri L, Kereiakes DJ, Yeh RW, Driscoll-Shempp P, Cutlip DE, Steg PG, Normand SL, et al. Twelve or 30 months of dual antiplatelet therapy after drug-eluting stents. N Engl J Med. 2014;371(23):2155-2166.

13. Mauri L, Elmariah S, Yeh RW, Cutlip DE, Steg PG, Windecker S, Wiviott SD, et al. Causes of late mortality with dual antiplatelet therapy after coronary stents. Eur Heart J. 2016;37(4):378-385.

14. Valgimigli M, Campo G, Percoco G, Monti M, Ferrari F, Tumscitz C, Zuffi A, et al. Randomized comparison of 6versus 24-month clopidogrel therapy after balancing antiintimal hyperplasia stent potency in all-comer patients undergoing percutaneous coronary intervention Design and rationale for the PROlonging Dual-antiplatelet treatment after Grading stent-induced Intimal hyperplasia study (PRODIGY). Am Heart J. 2010;160(5):804-811.

15. Colombo A, Chieffo A, Frasheri A, Garbo R, MasottiCentol M, Salvatella N, Oteo Dominguez JF, et al. Second-generation drug-eluting stent implantation followed by 6- versus 12-month dual antiplatelet therapy: the SECURITY randomized clinical trial. J Am Coll Cardiol. 2014;64(20):2086-2097.

16. Patti G, Cavallari I. Extended duration dual antiplatelet therapy in patients with myocardial infarction: A studylevel meta-analysis of controlled randomized trials. Am Heart J. 2016;176:36-43.

17. Friede $\mathrm{T}$, Rover $\mathrm{C}$, Wandel $\mathrm{S}$, Neuenschwander $\mathrm{B}$. Meta-analysis of two studies in the presence of heterogeneity with applications in rare diseases. Biom J. 2017;59(4):658-671.

18. Davey J, Turner RM, Clarke MJ, Higgins JP. Characteristics of meta-analyses and their component studies in the Cochrane Database of Systematic Reviews: a crosssectional, descriptive analysis. BMC Med Res Methodol. 2011;11:160.

19. Gonnermann A, Framke T, Grosshennig A, Koch A. No solution yet for combining two independent studies in the presence of heterogeneity. Stat Med. 2015;34(16):24762480 . 